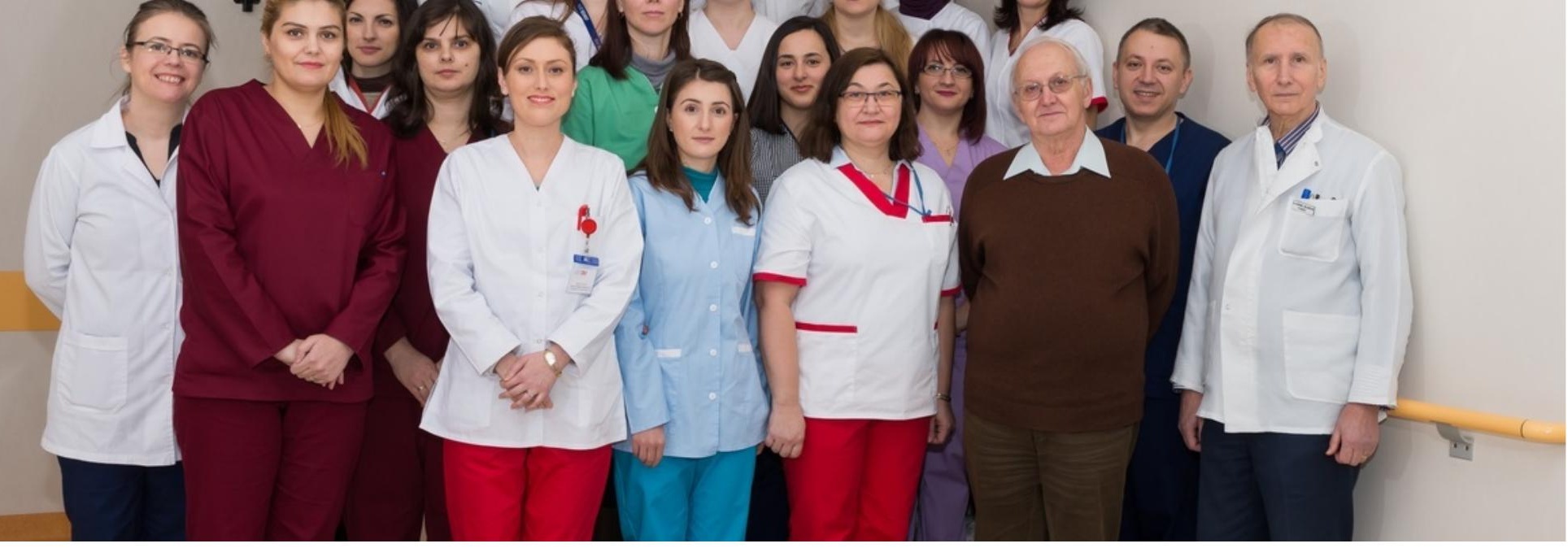

TRANSCEND a new Translational Medicine hub at the east edge of European Union.

$\begin{aligned} \text { Authors: } & \text { Eugen Carasevici } \\ \text { Submitted: } & \text { 31. December } 2017 \\ \text { Published: } & \text { 4. January } 2018 \\ \text { Volume: } & 5 \\ \text { Issue: } & 1 \\ \text { Languages: } & \text { English } \\ \text { Keywords: } & \text { Development, medicine, transcend } \\ \text { DOI: } & 10.17160 / \text { josha.5.1.376 }\end{aligned}$

Journal of Science,

Humanities and Arts 


\section{TRANSCEND a new Translational Medicine hub at the east edge of European Union.}

Romania is part of the string of countries that frame the eastern border of European Union, from the Baltic Sea to the Black Sea. The university city of Iasi is located at the eastern border, in the north-east of the country.

Regional Institute of Oncology (IRO) in Iasi, Romania is a new public health institution (active since 2012) with 300 beds, committed for cancer diagnostic and treatment, and covering the NorthEast part of Romania (roughly $44000 \mathrm{Km} 2$ and 5 million inhabitants). Main clinical services are surgery, medical oncology, hematology, radiotherapy, palliative care, medical imagistic department, pathology, and clinical laboratory. Starting from 2016 two new functional components are operational in the frame of IRO configuration: a Stem Cell Transplantation Service connected to the Hematology Clinic and a Center of Fundamental Research and Experimental Development in Translational Medicine (TRANSCEND). All TRANSCEND laboratories dedicated to research are included in a separate selfcontained building. As a consequence two interconnected research levels will make TRANSCEND a unique translational hub: molecular medicine and nuclear medicine. The molecular medicine field of research will be represented by a flow of chained activities developed in the frame of following units: Molecular Diagnostic Imaging (flow-cytometry and tissue digital cytometry), Cytogenetics and Molecular genetics, Molecular Biology (PCR, sequencing, microarray, molecular cloning), Cell culture and and cell manipulation (2D and 3D-organ-like cultures), Proteomics, cryogenics and biobanking, and Bioinformatics. The nuclear medicine level will consist of a radiotracer synthesis and research unit (cyclotron and radiopharmaceutical laboratories) and a PET-CT translational diagnostic unit.

The functional concept of TRANSCEND is equally in favor of independent continuation and subsistence of each unit through dedicated projects and financial capital as well as comprehensive projects that fit into place all units of the center for the design of a biomarker with diagnostic value and ultimately linked to the application of a specific and tailored therapy. Pairing Diagnostics with Therapeutics is currently framed under the notion of theranostics. TRANSCEND will promote this convention implying that drugs must be paired with diagnostic biomarkers to enable the right drug to be selected for the right patient at the right time. Central to the integration in such a scheme, which aspire to diagnose, deliver targeted therapy and monitor the response to therapy, is the growth of units of proteomics, bioinformatics and isotopic tracer production for developing a translational biomarker repertoire and a functionally interpretable systems medicine. 


\section{$\underline{\text { Molecular Medicine }}$}

\section{Molecular Diagnostic Imaging:}

Department of Flow Cytometry is equipped with four multi-laser cytometers, offering complex, multiparametric analyses within a very wide range of applications (immunophenotyping, cell cycle, viability, cell proliferation, soluble cytokine detection, DNA fragmentation): Aria III, BD Biosciences (3 lasers: 488/375/633 nm, 10 fluorescence detectors + one cell sorting platform), Navios, Beckman Coulter (3 lasers: 488/405/633 nm, 10 fluorescence detectors), Gallios, Beckman Coulter (3 lasers: 488/405/633 nm, 10 fluorescence detectors), Calibur, BD Biosciences (2 lasers: 488/ 635nm, 4 fluorescence detectors). In addition, the Aria III (Becton Dickinson) cytometer can be used to sort any cell population of interest, which can be stored/ used further for multi-disciplinary purposes (cell culture, FISH, PCR, microarray). Main routine laboratory investigations performed in this department are: - Lymphocyte Screening Test (one tube, 12 markers) • Panel for B-cell chronic lymphoproliferative disorders (four tubes, 31 markers)

- Panel for T-cell chronic lymphoproliferative disorders (six tubes, 44 markers) - Panel for NKcell chronic lymphoproliferative disorders (three tubes, 24 markers) - Acute leukemia orientation test (one tube, 8 markers) - B cell Precursor Acute Lymphoblastic Leukemia Panel (four tubes, 34 markers) - T cell Acute Lymphoblastic Leukemia Panel (four tubes, 32 markers) - Acute Myeloblastic Leukemia Panel (seven tubes, 56 markers) $\bullet$ Multiple myeloma tests $\bullet$ Post-Therapy Test (Minimum Residual Disease) - custom panel - Confirmation of pulmonary sarcoidosis (bronchoalveolar lavage) - Test to assess the degree of DNA fragmentation in spermatozoa. • Technical counseling and instrumental support in flow cytometry for external users.

Additionally, the development of databases containing normal and pathological references based on multiparametric immunophenotypic data files in malignant hematological diseases, as well as the building of a multidisciplinary network for diagnostic standardization (hematologyimmunology-oncology-histopathology) are underway. We expect that this will generate the premises for personalized therapy of patients with malignant hematopoiesis. The Molecular Imaging department also provides the tools to quantify the expression of target marker molecules in either histological specimens or life cell cultures, and integrate such data at ranges starting from subcellular (i.e. cell organelles) to organ fragment and tissue. Three microscopic systems, each with a certain level of informatic control, stand now at the core of this facility. A general pathology upright Nikon Eclipse E800 stand with filters for 3 very common fluorochromes, a large range of magnification objectives, and image capture assures convenient analysis of standard morphopathologial slides, or of tissue microarrays created by a semiautomatic Galileo TMA CK 4500 system. Fully computer-programmed acquisition and analysis of either conventional slides or of cell culture inserts are assured by an inverted Zeiss Observer Z1 platform that includes a motorized stage with 8 slide positions, culture gases $(\mathrm{CO} 2, \mathrm{O} 2)$ for inserts, more than 12 spectral channels for fluorochromes is based on a Tissue Gnostics software system. Subsequently, we deploy either single point (on clinical bioptic materials) analysis or time framed cell culture evaluation for samples that allow translational 
projects for oncological diagnostic markers (in both immunostainings and hybridization assays) and basic research programs - mouse system being our current model.

\section{Cytogenetics and Molecular genetics}

This department has a full-service laboratory which offers cytogenetic, molecular genetic, and fluorescent in situ hybridization (FISH) testing for acquired chromosome abnormalities (leukemia, lymphoma, and solid tumors). In addition, molecular testing is offered for selected single gene disorders. The cytogenetics lab conducts conventional chromosome banding techniques and molecular cytogenetic methods (fluorescence in situ hybridization) on bone marrow and peripheral blood cells samples of the patients with hematologic malignancies. The detection of chromosome abnormalities provides significant important information for making a precise diagnosis, supporting prognosis and risk stratification, and determining an appropriate treatment protocol. The department also works closely with the flow cytometry and molecular biology unit to supply the data needed to answer complex hematologic questions. A MetaSystems automated reading platform is used for fast, accurate highresolution analysis and greater objectivity and sensitivity. This results in the easier identification of chromosome abnormalities, lower turnaround time and fewer culture failures. Handling approximately 300 referrals per year, the department is one of the largest specialist cancer cytogenetics units in our country. The cytogenetics laboratory is accredited by Romanian Accreditation Association and also complies with professional standards issued by the European Cytogeneticists Association (ACGS) and participates in relevant EQA schemes (CEQAS).

\section{Molecular Biology}

The molecular biology unit acts as a core facility providing a wide array of services for high precision, high throughput analysis of DNA and RNA to support molecular biology projects within the Regional Institute of Oncology, Iasi, Romania.

Our Services Include Automated DNA Isolation, Genotyping, DNA Sequencing both Sanger and NGS, Identification of Copy Number Variation, SNP, and LOH, Quantitative Real-Time PCR, Training Services, Consultation Services.

Our Instruments Include ABI 3500 Genetic Analyzer, Beckman Coulter Biomek FX Automated Dual Arm Liquid Handler, NGS platforms ( Life Technologies IonTorrent and Illumina MiSeq), Agilent microarray platform, NanoDrop Spectrophotometer, Bioanalyser 2100 -Agilent, Qbit fluorometer, Diagenode Bioruptor Pico sonication device, LightCycler480II, Agilent Mx3005P qPCR System. We offer comprehensive services for Sanger Sequencing and Next Generation Sequencing. Employing two NGS platforms we cover a wide range of NGS applications including Whole Genome and Whole Exome Sequencing as well as RNAseq, Chromatin Immunoprecipitation Sequencing (ChIP-seq) and Targeted Sequencing. In addition, the lab hosts a range of state-of-the-art instruments and 
offers technical expertise for genome and gene expression analysis, including high-throughput Real-Time Quantitative PCR. The instrumentation allows fulfilling DNA/RNA extraction, quantification, reversetranscription, polymerase chain reaction (PCR), electrophoretic migration, multiplex ligation-dependent probe amplification (MLPA), Real-Time PCR, DNA sequencing, and microarray. Main routine diagnostic laboratory investigations performed in Molecular Diagnostic Department are: • Fusin Genes identification for Acute Leukemia: E2A-PBX1; MLLAF4; BCRABL (p190); SIL-TAL; PML-RARA; AML1-ETO; CBFBMYH11; FLT3 ITD, and FLT3 d835; NPM1

- Identification of K-RAS gene mutations - codon 12: Ala c.35G>C, Arg c.34> G, Asp c.35G>A, Cys c.34G> T, Ile c [34G> A, 53G> ], Leu c (34 G> C, 35 G> T), Ser c.34 G>A, Val c.35 G> T; Codon 13: Asp c. 38G> A, Cys c.37G> T; Codon 61: Arg c.182A> G, His C.183A> T, Leu c $182 \mathrm{~A}>\mathrm{T} \cdot$ Identification of N-RAS gene mutations - codon 12: Ala c.35G $>\mathrm{C}$, Arg c.34> G, Asp c.35G>A, Cys c.34G> T, Ile c [34G> A, 53G > ], Leu c (34 G> C, 35 G> T), Ser c.34 G> A, Val c.35 G> T; Codon 13: Asp c. 38G> A, Cys c.37G> T; Codon 61: Arg c.182A> G, His C.183A> $\mathrm{T}$, Leu c 182A $>\mathrm{T}$ - The identification of EGFR gene mutations (del exon 19; S768I; L858R; T790M; G719X (G719A, G719C, G719S) exon insertions 20) • Identifying the V600E mutation in the BRAF gene - Microsatellite instability identification (MSI): BAT25, BAT26, NR21, NR22, NR23 - Gene expression for ERBB2, ESR1, PGR and MKI67 (MammaTyper) markers from fixed and paraffin embedded probes. - NGS (Illumina platform) for 80 genes involved in the predisposition to oncological pathology (germline mutations). - NGS (Illumina platform) for BRCA1 / 2 genes. (Germline mutations). - CMV Viremia by Real-Time PCR • Viremia of Polyoma BK Virus by RealTime PCR $•$ HPV genotyping $\bullet$ Sequencing the TK domain of ABL1 gene in chronic myeloid leukemia $\bullet$ Cloning rearrangement of B cells - detection of clonal Ig heavy chain - Clonal rearrangement of $\mathrm{T}$ cells - identification of clonal rearrangements of TCR • Quantitative analysis of chimeras for bone marrow transplant patients: The tested STRs are: TH01 (11p15p15.5), FES / FPS (15q25-qter), CSF1PO (5p33.3-q34) Q21), vWA31 (12p13.312p13.2), D3S1358 (3p21). Detection of $\mathrm{t}(11,14)$ (q13; q32) (fusion between immunoglobulin heavy chain (IGH) gene and CCND1 proto-oncogene (bcl-1, PRAD1) The target region is the Major Translocation Cluster (Mantle Cell Lymphoma)

- Identification of BCL2 / IGH-t (14; 18), Major Breakpoint Region (MBR) and Minor Cluster Region (mcr) in $80 \%$ of follicular lymphomas (FL) and $30 \%$

\section{Cell Cultures}

Cell Cultures facility provides state-of-the-art services to support experimental demands ranging from routine culture techniques to advanced 2D and 3D cultures and spheroids. Fundamental differences in the cell culture environment compared with the cell microenvironment affect cell behavior. 
Two-dimensional (2D) cultures grown on plastic lack exposure to components of the extracellular matrix that are present in vivo, lack heterotypic cell-cell interactions and do not recapitulate threedimensional (3D) tissue architecture.

Adding the third dimension to a cell's environment creates significant differences in cellular characteristics and behavior. Importantly, these differences normally create a greater similarity between the cultured cells and tissues of a living organism (e.g. a human being) - leading to more useful data and more relevant research. Usually, cells are plated between 2 layers of matrigel together with the defined medium. Some cells become quiescent and selforganize into polarized, spheroidal acinar structures in 3DMatrigel matrices, while some other cells continue to proliferate, forming large disorganized colonies with stellate, invasive cell projections that reflect aggressive tumors.

Mammosphere cultures, which are distinct from the 3D matrigel (morphogenesis assay) provide an assay for self-renewal of cells. A specific density and number of cells will be plated in low adherence plates within a defined medium. These conditions prevent differentiation and allow selfrenewal of stem or progenitor cells.

Different inhibitors in human cell line can influence mammospheres formation. Mammospheres can be dissociated mechanically and enzymatically to single cells and placed in suspension culture, or collect them and put them on paraffin for future analysis.

Routine tissue culture needs are supported by two biosafety cabinets, a pair of $\mathrm{CO} 2$ incubators, refrigerators and freezers, environmental rooms, centrifuges, water baths, and inverted microscopes.

Methodology $\bullet$ 3D culture and coculture: Cells suspended in a matrix, such as a matrigel, to create a greater similarity between the cultured cells and the living organism (e.g. a human being). • Mammosphere assay: Mammospheres cultures which are different than 3D culture, provide an assay for self-renewal of cells. A specific number of cells will be plated into ultralow adherent plates with the defined medium. Those conditions prevent differentiation and allow self-renewal of stem or progenitor cells. - In vivo imaging technology (animal experiments): Vevo® 2100 System (Regional Institute of Oncology, Transcend Centre under an agreement with CEMEX research center of Gr.T.Popa University of Medicine and Pharmacy, Iasi) provides quantification of the target biomarker.

\section{$\underline{\text { Proteomics and Imaging mass spectrometry core facilities }}$}

Mass spectrometry (MS) unit at TRANSCEND - IRO, Iasi acts as a core facility that brings together innovative mass spectrometric-based technologies. The mission of the MS unit is to perform fundamental and applied studies in proteomics and molecular imaging as a part of interdisciplinary research programs within our center and researchers from all over the world. MALDI - Imaging investigates cell structures at a macromolecular level. Inside human and 
laboratory animals cells, proteins are working together in complex structures and are responsible for different biological and pathological processes. To understand the working mechanisms of protein complexes, three-dimensional imaging of normal and diseased tissues is essential. Main research aim for the coming years is to develop and apply mass spectrometry-based approaches as prognostic and diagnostic tools. This will be an important step for establishing more effective treatments in personalized medicine.

Workflow and applications:

Protein identification from complex mixtures (biological samples) - biomarkers Following chromatographic separation using small samples volumes (microliter range) and automatic fraction collection and deposition on MALDI plates, protein identification is carried out either using a top-down approach (spectra acquisition in reflector mode with in-source decay - reISD) or a bottom up approach (proteolytic digestion followed by LC-MALDI-MS/MS). Tissue molecular phenotyping Mass spectrometric biomarker identification is carried out directly on tissue sections, thus allowing access to the complete spatial and histological information of different macromoleculs (peptides, proteins and lipids). MALDI Imaging of cancer tissue specimens allows for identification of novel protein biomarkers and investigation of molecular mechanisms of local invasiveness and resistant to chemotherapy. Metabolomics Spatial distribution of endogenous metabolites is investigated via mass spectrometric imaging and allows the creation of tissue metabolomics profiles.

\section{Infrastructure:}

- MALDI-TOF/TOF Mass Spectrometer (ultrafleXtreme TM, Bruker Daltonics) - Rapid Separation LC nano System (Dionex UltiMate 3000, Thermo Scientific) • LC MALDI Fraction collector (Proteineer fc II, Bruker Daltonics) • Cryostat for MALDI Imaging (CM1860 UV, Leica) - Matrix Deposition Device for MALDI Imaging (ImagePrep, Bruker Daltonics)

\section{Bioinformatics}

Research focuses on the study of the structure - dynamics - activity relationship of biologic molecules/macromolecules in order to get insight into the altered molecular mechanisms responsible for various types of cancer. Experimental techniques such as NMR, FRET X-ray crystallography, and many other biophysical techniques can elucidate many aspects of structure and dynamics of biomacromolecules but their limitations reside in sensitivity, applicability and accessible time scales. In our research, we use theoretical modeling at different levels of accuracy including ab-initio and DFT calculations, molecular docking, homology modeling and molecular dynamics simulations, to obtain details that are not available to the experiment. The topics addressed encompass modeling of dynamics of 
proteins and protein complexes, target protein-drug interaction prediction for therapy optimization, prediction of functional consequences of molecular alterations and comparative modeling of unresolved protein structures. The hardware infrastructure consists of a HighPerformance Computing (HPC) system containing GPU equipped servers and 48 TBytes of storage delivering 9.84 TFLOPS of computing power. For data analysis and interpretation, graphical workstations are available.

\section{Cryogenic Biobanking Core}

The newly cryogenic storage area is a state-of-the-art cryogenic repository facility located both inside the TRANSCEND building and within Stem Cell Facility of the Regional Institute of Oncology. Biological specimens are stored in high capacity vapor phase liquid nitrogen cryogenic storage tanks. Currently, 3 cryogenic units, by Taylor Wharton, are maintained with 24-hour, 365day security and daily temperature monitoring. The capacity of these units ranges 70,000 vials or transfusion bags. The biobanking facility will make possible to preserve the most diverse collection of cell lines and DNA available for diverse experimental studies. In addition to these high-quality biospecimens, it will be possible also to maintain tissue, plasma, serum, urine, and cerebrospinal fluid.

\section{$\underline{\text { Nuclear Medicine }}$}

\section{Radiopharmaceuticals unit}

The nuclear medicine level will consist of a radiotracer synthesis and research unit (cyclotron and radiopharmaceutical laboratories) and a PET-CT translational diagnostic unit. Medicinal radiocompounds to be produced are both fluorodeoxyglucose (FDG) and some others radiotracers by using different radioisotopes with short half-lives (like 18F, 11C, 13N) eventually coupled with pharmacologically active molecules. Radiopharmaceutical laboratories network consists of different areas. An unclassified area is composed of spaces for unprocessed materials quarantine, Heating, ventilation and air conditioning (HVAC), Cyclotron (Self Shielded) zone and Cyclotron control room, two places to stay for personal. The classified experimental area is consisted of: • Two Hot Labs, one for FDG production and one for research, holding two Hot Cells providing each a safe containment box in which the required equipment can be controlled and manipulated. - A quality control (QC) laboratory, equipped with: - Gas chromatograph by flame ionization detector (Bruker GC436); • Highperformance liquid chromatography (Thermo Scientific), equipped with electrochemical detector (Thermo Scientific) and radiochemical detector (Carroll \& Ramsey 105S-1 single channel radiation detector); - Thin layer chromatography scanner, equipped with radiochemical detector (Carroll \& Ramsey EZ-Scan TLC System); - Gamma spectroscopy (Canberra Gamma Spectroscope with Osprey MCA System); • Dose calibrator (Capintec CRC@-55t Radioisotope Dose Calibrator); • Wipe test counter (Capintec Caprac®-T Well/wipe test counting system); • pH-meter (METTLER TOLEDO SevenExcellence ${ }^{\text {TM }}$ S400 
pH meter); - Endotoxin test system (Charles Rivers Endosafe ${ }^{\circledR}$-PTS Portable Endotoxin Test System); • Millipore Direct-Q® 3 UV water purification system; • Analytical balance (Mettler Toledo XS204) • Fume hood with laminar air flow. • A microbiology laboratory, equipped with: • Gram Biocompact RR 410 LH refrigerator; • Genlab INC/50/SS/F/DIG/DD incubator; • Genlab INC/150/SS/F/DIG/DD incubator; • two Tuttnauer tabletop 2340E autoclaves. • A raw material preparation room, equipped with: • Gram Biocompact RR 410 LH refrigerator; • Gram Biocompact RF 410 LH freezer, • Also rooms for labeling, distribution, waste material quarantine, shower and for cleaning materials.

Clinic molecular imaging and PET-CT translational diagnostic unit.

Operation in this department is headed for providing state-of-the-art clinical services in nuclear medicine and to be a leading resource for education and research based on advanced nuclear imaging techniques. It is based on very advanced equipment: these include one gamma camera, which is a dual-head single photon emission computed tomography (SPECT) system and a positron emission tomography (PET) with integrated CT (PET/CT) scanner combining a dedicated full ring PET/CT tomography and a multidetector CT unit. An integrated computer network and PACS system with PACS viewing capabilities and clinical workstations are available. The department has the ability to deploy a full spectrum of general nuclear and molecular imaging exams, from bone and thyroid scans to gated myocardial perfusion scintigraphy, and myocardial viability assessments. PET/CT clinical and research imaging is mainly oriented to oncological studies for monitoring of responses to therapy of various cancers including lymphoma, colorectal, and lung.

Integrative animal research - external collaboration

TRANSCEND has an ongoing institutional collaboration with CEMEX (The Center for Research and Development in Experimental Medicine) of Grigore T. Popa University of Medicine and Pharmacy Iasi. $\quad$ CEMEX is a state of the art facility for animal research, fully equipped for rodent and large animal housing and husbandry, continuous telemetric monitoring of biological variables and imaging solutions. Selected available infrastructure: - Complete individually ventilated cage system for housing of mice (Sealsafe Next System ${ }^{\circledR}$ - Tecniplast Italy) - total housing capacity for 2000 mice and 1000 rats • Ultra-high frequency Ultrasound Imaging platform with Linear Array Technology - Vevo @ 2100 with color and power Doppler mode for tumor blood flow quantification, 3D Mode Imaging and Volume Analysis for longitudinal assessment of implanted tumor growth. - Integrated in-line preclinical whole-body PET/MRI for rodents (nanoScan, Mediso Medical Imaging System) - Complete surgical suites for small and large animals

Highly qualified staff complements the technical endowment and generate an active and competitive environment. The new research center is ready to respond to those seeking collaboration across the European space and to promote the development of biomedical exploration across the eastern border of the Union. 


\section{Eugen Carasevici}
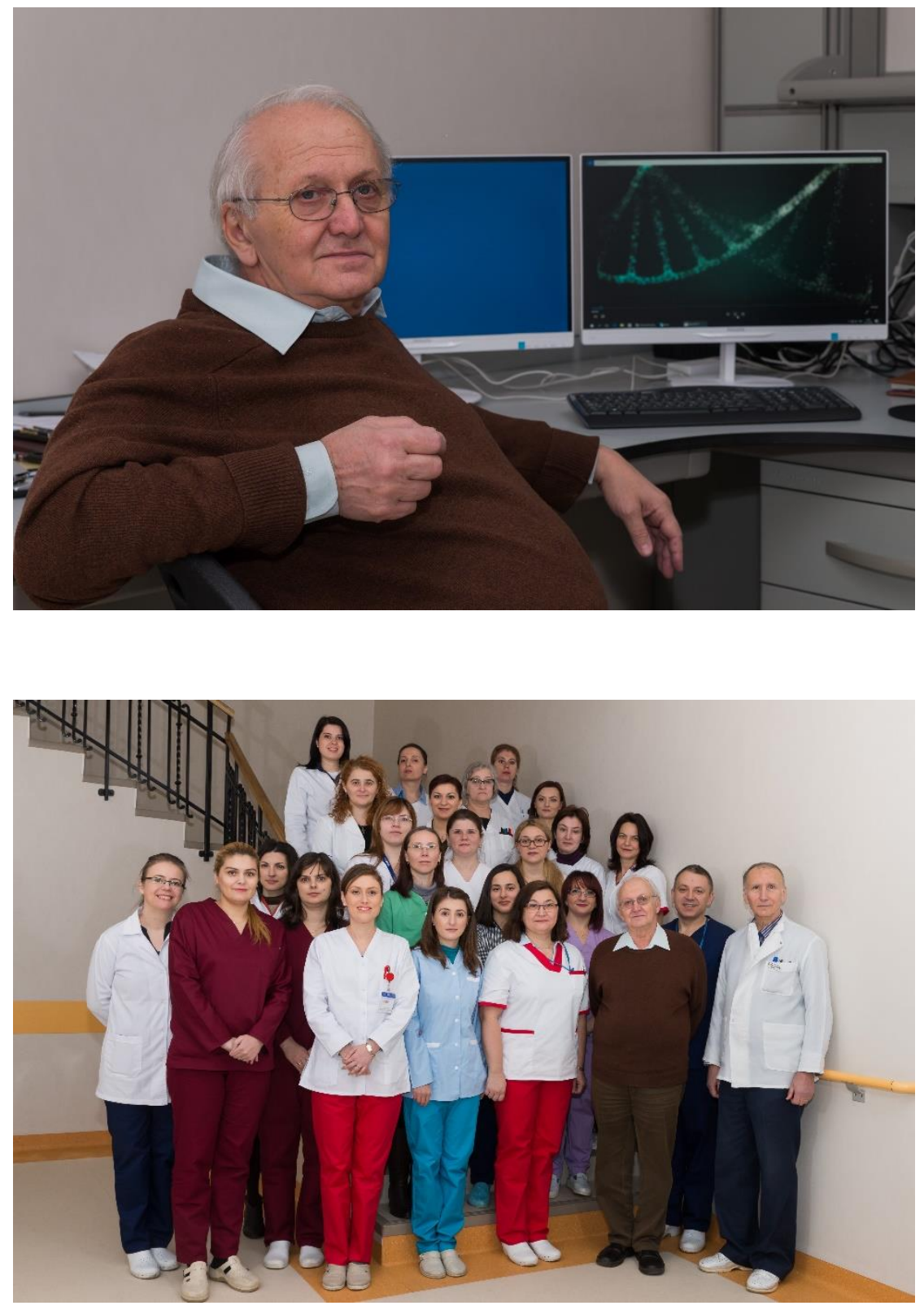

TRANSCEND Research Center

Regional Institute of Oncology (IRO), Iasi

2-4 General Henri Mathias Berthelot

Iasi 700483, Romania

Mail: eugen.carasevici@gmail.com 


\section{EUGEN CARASEVICI, 10 March 1945, Garbova, Alba County, Romania.}

Present position: Senior Research Consultant at TRANSCEND (Center of Fundamental Research and Experimental Development in Translational Medicine), Regional Institute of Oncology (IRO), 2-4 Henri Mathias Berthelot, 700483 Iasi, Romania.

Professional development: graduate of the Faculty of Medicine 1969, Gr.T.Popa University of Medicine and Pharmacy Iasi, Romania, clinical internship 1969-1971, residency in Radiology and Radiotherapy 1971-1974, Radiology-Radiotherapy specialty 1974, senior consultant RadiologyRadiotherapy 1990, Oncology-Chemotherapy Second Competence 1982, OncologyChemotherapy specialty 1987, senior consultant Clinical Immunology 2000, overspecialization Laboratory Immunology 2004; DAAD Scholarship, Heidelberg, Germany, German Center for Cancer Research 1975-1976, PhD in Medicine 1983 (Relationship between Radiobiological Effect and Antitumoral Immunity in Experimental Cancer). Assistant Professor Radiology-Radiotherapy (1973-1987); Associate professor of Immunology 1992-1995; Professor for Immunology 19952010; PhD coordinator 1999-2014.

Achievements: Introduction of chemotherapy at Radiology-Oncology clinic, St. Spiridon University Hospital, Iasi (1972); Award of the National Immunology Commission 1981; Foundation of the Laboratory of Tumor Immunology (1986), Clinic of Radiology-Oncology, Foundation of the Immunology Department Gr.T.Popa University of Medicine and Pharmacy, Iasi (1992), Organization of two International Immunology Courses (1992 and 2000); Reconfiguration and extension of the Tumor Immunology Laboratory, renamed as Immunology and Genetics Laboratory (2002); Foundation at Gr.T.Popa University of Medicine and Pharmacy in Iasi of an Interdisciplinary Molecular Medicine Platform on the basis of a national grant of 1.2 million Euros (2006); Vice-president of the Center "Biomedical Research Center Gr. T. Popa" and coordinator of oncological research department; Head of Compartment of Molecular Biology, Regional Institute of Oncology Iasi from 2012, Winning the funding competition for TRANSCEND research center (Fundamental Research and Experimental Development in Translation Medicine), at Regional Institute of Oncology Iasi (13 millions Euros European funds); Ability to generate work teams, to formulate goals and polarize competencies. Coordinating 17 research projects funded by national or European bodies, $14 \mathrm{PhD}$ theses, author and co-author of 111 publications, member of seven scientific societies, member of 7 editorial groups.

Languages spoken in order of competences: French, English, German.

Mail address: eugen.carasevici@gmail.com 